\title{
A modeling approach towards improving compliance of treated water quality to reduce manpower and chemicals
}

\author{
Alaa Husaeen Wadie \\ Head of Environmental Eng. Dept. /College of Eng. /Babylon University \\ Email address: \\ dr_ahw@yahoo.com

\section{To cite this article:} \\ Alaa Husaeen Wadie. A Modeling Approach towards Improving Compliance of Treated Water Quality to Reduce Manpower and \\ Chemicals. International Journal of Environmental Monitoring and Analysis. Vol. 1, No. 5, 2013, pp. 194-202. \\ doi: $10.11648 /$ j.ijema.20130105.15
}

\begin{abstract}
In water treatment processes, the individual unit operations are complex, non-linear and poorly understood. Whilst many models have been developed to improve process understanding, these are rarely in a form easily exploited by the control engineer. Attempts to improve the performance of water treatment works through the application of improved control and measurement have had variable success. This paper discusses investigations into the application of feed forward control on the clarification process of a small-scale pilot plant. The application aimed towards maximizing the efficiency of the chemical coagulation process. To achieve this, a simple computer program written in Visual Basic version 6 models to a chief the process operating conditions. Mathematical models based on historical plant data covering 18 months analyzed by stepwise multiple regression analysis. The following parameters were important determinants of coagulant dose and $\mathrm{pH}$ control reagents: river turbidity, $\mathrm{pH}$, temperature, total dissolved solids, and plant flowrate. A predictive equation developed from the data, of the form: $\mathrm{Al}_{2}\left(\mathrm{SO}_{4}\right)_{3}(\mathrm{mg} / \mathrm{L})=\mathrm{a}^{*} \mathrm{Q}+\mathrm{b}^{*} \mathrm{Turb}+\mathrm{c}^{*} \mathrm{TDS}+\mathrm{d}^{*} \mathrm{pH}+\mathrm{e}^{*} \mathrm{Temp}+\mathrm{f}$. The aim of this model is to provide water treatment operators with a tool that enables prediction of chemical reagents and treatment conditions for selected removal of turbidity, based on raw water quality data. While for adjusting $\mathrm{pH}$, whether lime or soda ash are added, the pretreatment of water supplies involves the use to decrease the acidity, to soften, and to clear drinking water, calcium oxide $(\mathrm{CaO})$, commonly known as quicklime or burnt lime. The addition of lime is with the form: $\mathrm{CaO}(\mathrm{mg} / \mathrm{L})=\mathrm{j}+\mathrm{k} * \mathrm{pH}$. And for soda ash sodium percarbonate $\mathrm{Na}_{2} \mathrm{CO}_{3}$ the addition form is: $\mathrm{Na}_{2} \mathrm{CO}_{3}(\mathrm{mg} / \mathrm{L})=\mathrm{m}+\mathrm{n} * \mathrm{pH}$. The advantages of software program are significant in the operation of water treatment plant. The program designed as an aid, so the user can still customize and optimize the computer suggested design. Users are able to move forward in adjusting or optimizing the design in minutes, which is difficult for manual system. This system was an initial system, many new features and functions have to be added to the program to enhance the functions and make it commercially robust. It concluded that this system is very powerful tool in improving compliance of treated water quality to reduce labor and chemicals and to facilitate the organizations and individuals with better understanding on how their actions can have a direct impact on the treatment.
\end{abstract}

Keywords: Coagulation, Feed Forward Control, Model Definition, Turbidity, PH, Temperature, Total Dissolved Solids, Plant Flowrate

\section{Introduction}

Coagulation process is the traditional water purification process of the more important aspect, accurate control of dosing volume is good coagulation effect of the most important prerequisite. Coagulant filling volume automatic control is a difficult problem to solve, because it is not only with the parameters of water quality and quantity parameters, but also with clean water structures, performance and effectiveness of such self-coagulant factors [Fu-Yi Cui, Yuan-zhen, 1999]. Conventional treatment at large scale water treatment plants involves the use of inorganic coagulants to remove turbidity and color, and more recently to maximize removal of organic compounds. Maximizing removal of organic matter using inorganic coagulants impacted by the character and concentration of the organics, the turbidity, and alkalinity of the raw water. Removal of organics also influenced by the type of coagulant used its dose rate and the $\mathrm{pH}$ at which coagulation occurs [Zhong Chun-Chang, et al., 1989; Song Qi-min, et al., 1999; Chang, 2002].To date, few attempts 
have been made to model the relationships between raw water quality parameters and the use of coagulants and $\mathrm{pH}$ control reagents for removal of organics, color and turbidity. The treatment of surface waters for public supply generally involves coagulation to remove turbidity and color prior to disinfection. The correct coagulant dose and optimum $\mathrm{pH}$ value depend on the nature of the raw water and dosing rates will need to be adjusted from time to time as water quality changes; good control of coagulation conditions is essential for maintenance of satisfactory treated water quality and economic plant operation. Poor control leads to wastage of expensive chemicals, failure to meet water quality targets and less efficient operation of sedimentation and filtration processes. Good control can reduce labor and chemical costs and improve compliance with treated water quality targets. In addition, it permits more effective use of downstream processes and the cost of maintenance can be reduced as a result of less fouling [Yang people, 2000; Kenichi Kurotfanim, et al., 1995; Zhang Weiguo, 2000; Zhang, et al., 2007].

The main objective of the work described herein has been to evaluate, refine and automate the most effective methods of coagulation control and to promote their use within the Iraqi surface water and ground water. This work has involved laboratory and small-scale' pilot studies as well as data gathered from recording full-scale-water treatment plant.

\section{Problem Description}

\subsection{Overview of Water Treatment Operations}

Water treatment involves physical, chemical and biological processes that transform raw water into drinking water. However, contrary to most industrial processes, for which the quality of the input raw material is under control, the quality of the given raw water source may fluctuate due to natural perturbation or occasional pollution.

\subsection{Coagulation Control}

Coagulation is one of the most important stages in surface water treatment, allowing for the removal of colloidal particles. The main difficulty is to determine the optimum quantity of chemical reagent related to raw water characteristics. Poor control leads to wastage of expensive chemicals, failure to meet the water quality targets, and reduced efficiency of sedimentation and filtration processes In contrast, good control can reduce labour and chemical costs and improve compliance with treated water quality targets. The traditional method of controlling coagulant dose, called the jar-test, relies heavily upon human intervention. It involves taking raw water samples and applying different quantities of coagulant to each sample [Lind, 1994].

After a short period, each sample assessed for water quality and the dosage that produces the optimal result used as a set point. Operators change the dose and make a new jar test if the quality of treated water changes. Disadvantages associated with such a procedure are the necessity to rely on manual intervention, and lack of adaptation to abrupt changes of water characteristics. More recently, an automatic device, called a streaming current detector (SCD) [Bernazeau, et al., 1992; Dentel, 1995] has emerged. This device based on the measurement of the net residual charge surrounding turbidity and colloidal particles in water. It requires a set point tobe entered, assumed to represent an optimum water quality standard. Streamingcurrent values above the set point reveal an excess of coagulant, while values below the set point indicate insufficient coagulant dosage for full flocculation to occur. A jar test needs to be carried out to determine the set point. Disadvantages associated with the SCD are its operation cost and the limited efficiency of certain types of raw water quality.

The objective of this paper is to propose an alternative to the jar-test and SCD methods allowing for the automatic determination of optimal coagulant dose from raw water characteristics, using an artificial network approach. This approach requires the availability of on-line water quality measurements at an upstream survey station. An important objective of the present study is to validate automatically the sensor measurements performed at the survey station as to provide reliable inputs to the automatic coagulation control system.

This paper addresses the problem of building software for on-line determination of optimal coagulant dosage from raw water characteristics such as river turbidity, $\mathrm{pH}$, temperature, total dissolved solids, and plant flow rate. Previous studies [Mirsepassi, et al., 1995; Evans, et al., 1998; Grimm, and Lynch, 2004] have shown the potential effectiveness of such an approach based on artificial neural networks.

In the present system, such robustness is achieved using a modular composed of a pre-processing level responsible for prediction involving the determination of the optimal coagulant amount from raw water characteristics (Fig. 1).

\section{Automatic Coagulation Control System}

There are various types of automatic coagulation control systems. Numbers of basic control systems are available for these types of application are-

- $\quad$ Feed Forward

- Feed Forward with Feed Back trim

- $\quad$ Feed Back e.g. Streaming Current Detector (SCD).

The system developed in this study based on the Feed Forward principle. The automatic coagulation control system contains:

- The input parameters

- The output parameters

- Potential future developments The overall design of the system 


\subsection{Input Parameters (Feed Forward)}

- Raw Water quantity (flowrate), Q.

- Raw Water Turbidity, Turb.

- Raw Water Temperature, Temp.

- Raw Water $\mathrm{pH}, \mathrm{pH}$.

- Raw Water total dissolved solids, TDS.

\subsubsection{Function of the Inputs}

- Raw Water Quality, Quantity and Turbidity used to determine the basic coagulant dose level and, in combination with the coagulant dose, determine clarifier desludging intervals.

- Raw Water Temperature also affects the optimum coagulation.

- Raw Water Total Dissolved Solids affects the optimum coagulation.

\subsection{Output Parameters (Feed Forward)}

- Required coagulant dose rate setpoint $(\mathrm{mg} / \mathrm{L})$.

- Actual coagulant dose rate $(\mathrm{L} / \mathrm{hr} \& \mathrm{mg} / \mathrm{L})$.

- Required coagulation $\mathrm{pH}$ setpoint.

- Actual coagulation $\mathrm{pH}$.

- Clarifier de-sludge intervals (hr).

\subsubsection{Control System Specifications}

- Raw Water online turbid meter.

- Coagulation Control pH meters.

- The coagulant dose control function split between the main PLC and the

PLC/PID controller to allow "dial-dose".

- Optimum coagulation $\mathrm{pH}$ control may either be via a lookup table or an algorithm depending on site specific criteria.

- Coagulant dose algorithm.

Multi-variable parameter model based on the feed forward combined coagulation control system that can respond quickly to the raw water quality and quantity parameters, but the operation of the system depends on accurate and reliable instrument for each piece of work, the control system block diagram shown in Fig. 1.

\subsubsection{Control Systems}

Control systems can be considered to be of one of two types; feed-forward and feed-back. Feed forward control consists of measuring one or more properties of the input (raw water) and using this information to determine and maintain the required coagulation conditions. Due to the highly confidential nature of this work some details will remain hidden but the basic structure is detailed in Fig.1.

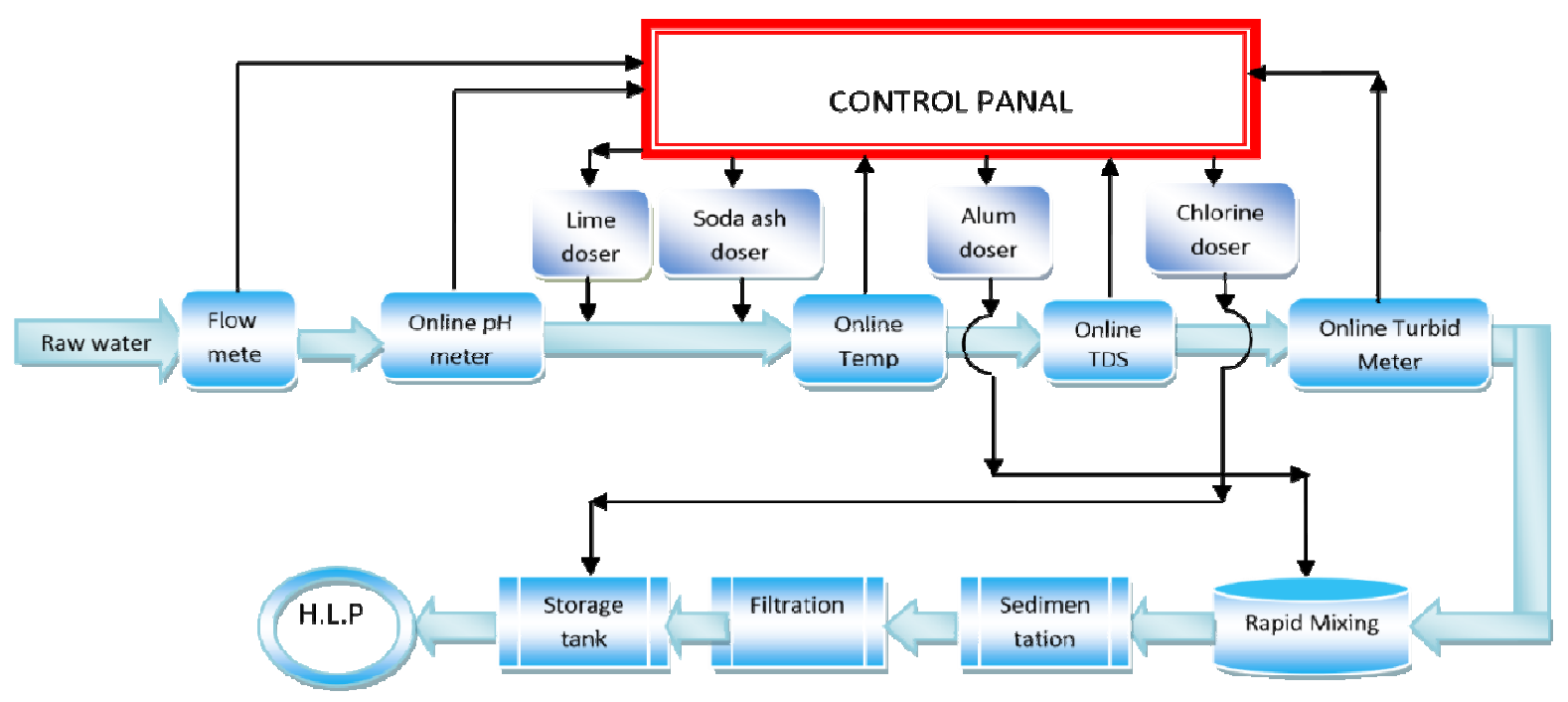

Fig.1: Schematic diagram of coagulation control system.

\subsection{Feed Forward Regression Based Coagulation Control}

The prediction of optimal coagulant dosage from water characteristics is a non linear regression problem. This approach is known to provide estimates of the conditional average of the output variable (here, the optimal coagulant dosing rate); given the observed values of the input variables (the raw water quality parameters).

Table 1: Statistical summary of raw water conditions for 18 months of study period, (Babylon Water Directorate, 2012).

\begin{tabular}{llllll}
\hline Parameter & Turbidity, $\mathbf{N T U}$ & $\mathbf{p H}$ & Temperature, ${ }^{\mathbf{0}} \mathbf{C}$ & Total dissolved solids, $\mathbf{m g} / \mathbf{L}$ & Plant flowrate, $\mathbf{~ m}^{\mathbf{3}} / \mathbf{h r}$ \\
\hline Maximum & 42 & 8.03 & 36 & 1140 & 600 \\
Minimum & 10 & 7.48 & 9 & 502 & 50 \\
Average & 16.3 & 7.68 & 21.8 & 715 & 160 \\
Stand. Dev. & 11.8 & 0.428 & 10.9 & 229.9 & 85 \\
\hline
\end{tabular}


Many water treatment plants acquire and archive large amount of data, primarily to satisfy regularity requirements. For some time now, these data sets have been used to develop deterministic and stochastic models that have been exploited for improving the efficiency of the dosing law [Cox and Graham, 1994; Adgar, and Cox, 1997; Prakash, 2004]. These methods estimate coagulant dose level from correlations with physical parameters describing the influent conditions of the plant. A major problem for investigators pursuing this route is the choice of the 'best' inputs. The choice of inputs to use in the feed forward law is an important design factor. Using a lot of inputs will generally improve model performance. However, including too many inputs increase the possibility of ill-conditioning and the associated model bias. Additionally, too many inputs leave the model susceptible to the possibility of individual sensor Failure. The study to be performed here are based upon linear techniques, therefore, to maximize the effectiveness of the methodology, several nonlinear transformations were performed on the input variables. Feed forward control uses measurements of raw water quality (for example, flowrate, turbidity, temperature, $\mathrm{pH}$, and total dissolved solids) to select coagulation conditions. This is done by relating historically successful coagulation conditions to raw water quality to produce one or more algorithms for selecting coagulant dose (and possibly $\mathrm{pH}$ ) on the basis of raw water qualify. Feed forward control is therefore dependent on the availability of detailed records of raw water quality and coagulation conditions covering the full range of water quality likely to be encountered (therefore data covering at least one full annual cycle is normally essential). This approach makes the implicit assumption that the historical plant doses were optimum. Feed forward control was studied in a small-scale pilot plant. This plant treats directly abstracted river water (approximately $3.75 \mathrm{~m}^{3} / \mathrm{m}^{2} . \mathrm{h}$ ) by coagulation with alum and a gravel filter as a sedimentation followed by rapid gravity filtration. The river quality does not change rapidly, the turbidity rise from less than $10 \mathrm{NTU}$ in dry weather to over 40 NTU in a wet weather.

Historical plant data covering 18 months was analyzed [Babylon Water Directorate, 2012] by stepwise multiple regression analysis. The following parameters were important determinants of coagulant dose: raw water flowrate, turbidity, $\mathrm{pH}$, temperature, and total dissolved solids. A predictive equation was developed from the data, of the form:

$\mathrm{Al}_{2}\left(\mathrm{SO}_{4}\right)_{3}(\mathrm{mg} / \mathrm{L})=\mathrm{a}^{*} \mathrm{Q}+\mathrm{b} *$ Turb $+\mathrm{c}^{*} \mathrm{TDS}+\mathrm{d}^{*} \mathrm{pH}+$ $\mathrm{e}^{*} \mathrm{Temp}+\mathrm{f},\left(\mathrm{R}^{2}\right)=0.758$ Where: $\mathrm{f}$, a to e, are empirical constants.

The control system is implemented on a computer (control panel) connected by many ports to an intelligent data loggers which were interfaced to instruments and pumps on the plant (Fig.1). The control program runs continuously and reads data from the data logger calculates $\mathrm{pH}$ and sends this value to the pump via the data logger.
Also read flowrate, temperature, $\mathrm{pH}$, turbidity and total dissolved solids. The control program monitors the final water turbidity aid trims alum dose as necessary to achieve the desired final water quality. Plant data is printed and displayed on a plant mimic diagram on the computer. The program can also support control of polyelectrolyte dosing. Normal or high rate depending on river water turbidity and interactive communication with the operator. Simplified diagram of feed forward control is illustrated in Fig. 2.

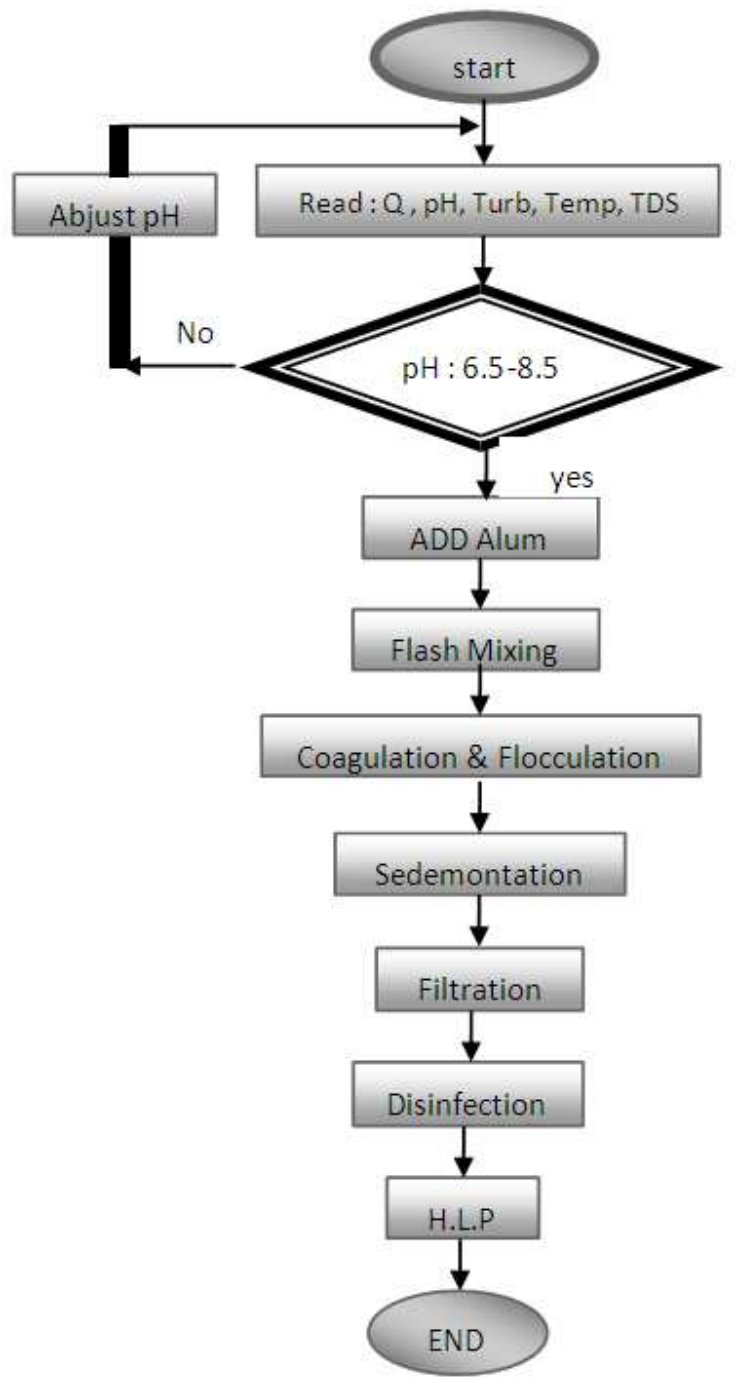

Fig. 2: Simplified diagram of feed forward control logic.

Computer modeling was used to assess the ability of the control system to control coagulant dose. A program was written containing a simple model of a clarification plant together with the control system. The clarification plant model enabled the final water quality to be calculated as a function of time under given raw water and dose conditions. By varying the raw water quality at random during the course of a computer run, a picture was built up of how the control system would function in practice. The results obtained over many simulations, runs have shown that the system is effective in controlling coagulant dose, and will 
maintain stable control even during periods of varying raw water quality.

Computer system, data acquisition parameters automatically, and according to the model the amount of automatic filling. This model is part of the former feedforward model. To improve control accuracy, stability, water quality, this requires further in order precipitating the establishment of water turbidity.

\subsection{Program Development}

The program developed by the author, using Microsoft Visual Basic (VB) 6.0 programming language, can assist users and operators of water treatment plants. Users can enter necessary information, such as flow rates and other water quality parameters. Then, the program calculates and provides information based on the inputs, instead of presenting the coding of the whole program, this paper mainly focuses on showing how a computer program could assist human operation in the treatment process. As typical coagulant doses, the program user firstly inputs the requested information via the interfaces of the system, then reviews and revises the adjustment created by the computer.

\subsubsection{VB Programming Language and Its Features}

The main reason VB is so popular and powerful is the success of the Windows operation system of the 1990s'. It has developed into a powerful development tool and supports implementation of complicated programming tasks. Compared with most other languages, VB is excellent with its graphical and user-friendly interface.

\subsubsection{Boundary Classes Needed in the Program}

The Information Request Interface, requests the user to input necessary information for calculation, such as flow rates, turbidity, $\mathrm{pH}$, etc. The Information Display Interface displays calculation results for the user such as calculated alum, lime, soda ash dose, expected sludge, etc.

\subsubsection{Control Classes in the Program}

Information Processor and Calculator is the core part of the whole system. It comprises all the calculation and information process programs. Almost all information, either inputted by users or to be displayed is processed by it. The basic functions of control class of the system include: data and information collection, boundary checking, database, and alum modeling and dose calculations. The smallscale pilot plant was used as an application site for this study. This scale plant has a nominal capacity to process approximately $3.75 \mathrm{~m}^{3} / \mathrm{m}^{2} . \mathrm{h}$. Several raw water parameters are measured on-line (Fig. 2), and jar-tests are performed quite frequently, providing the necessary target values (the optimal dosing rates to be estimated from water quality data).

The raw database consisted of 18 months measurements of five input variables (river turbidity, $\mathrm{pH}$, temperature, total dissolved solids, and plant flowrate). Simple descriptive statistics of the data are given in Table 1. Note that this data set, which represented all the available data at the beginning of this study, covers a period of one and half year and so can be expected to account for seasonal variations of water quality.

It is obvious, however, that periodic retraining of the system will be needed to ensure that the full range of water quality conditions (in the absence of dramatic pollution events) are accounted for, and to allow continuous adaptation of the system to any possible long-term evolution of water quality.

For program software operation the sequences could be divided into the following steps:

Step 1: user inputs program information via interacting with data request user interface. In this step, the basic information is inputted and ready to be processed.

Step 2: information processor and calculator then process the inputted information and perform calculations, modeling based on the dosing roles.

Step 3: software then saves the results in a temporary database. All the results gained from step 3 are stored in a temporary database, where the user can check and modify until the final decision is made.

Step 4: data display; the results from the information processor and calculator are displayed on the display interface, so the user could review and perform modifications or adjustments. The processes from 1 to 4 may be repeated if the user needs to make adjustments and modification.

Step 5: Once a proper dose is finalized and accepted by the user, the user may activate the model creator which is enhanced VB program. Both the original data inputted and the processed results will be used.

\subsubsection{Information Processor and Calculation Class}

As the core class of the software, Information process and calculation class includes all the modeling and calculations such as raw water quantity and quality modeling and calculation, alum and $\mathrm{pH}$ adjustment modeling.

\subsection{Implementation Process}

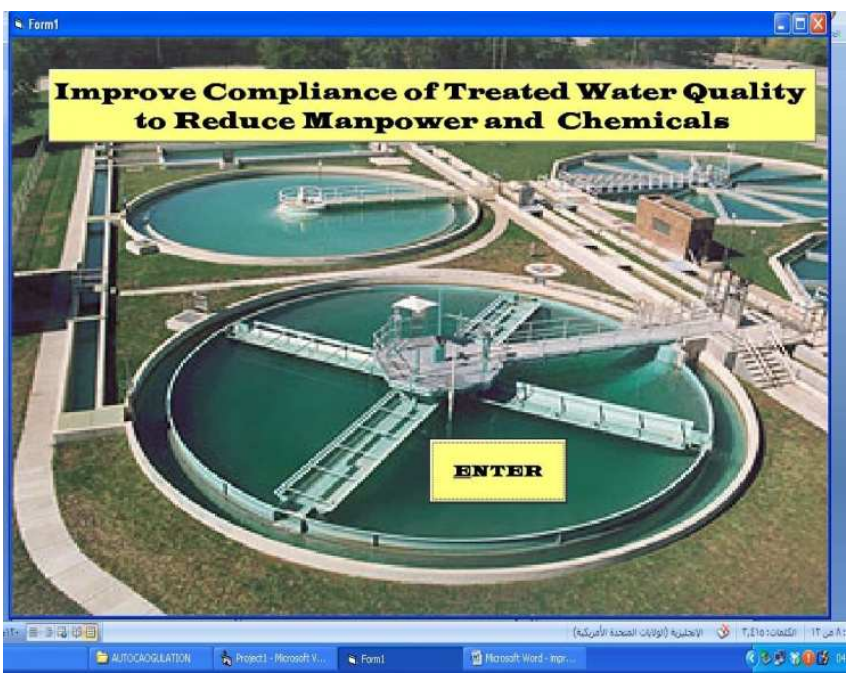

Fig.3: VB implementation diagram. 
Based on the requirement, analysis and design models established, Microsoft VB 6.0 programming targets the models. Fig. 3 shows the implementation diagram of the software. Approximately $8 \sim 15$ pages of VB programming codes were established to perform the preliminary design control system (See Appendix). The whole programming was stored in VB forms and modules. The major interfaces of screen shots are shown in Fig.4.

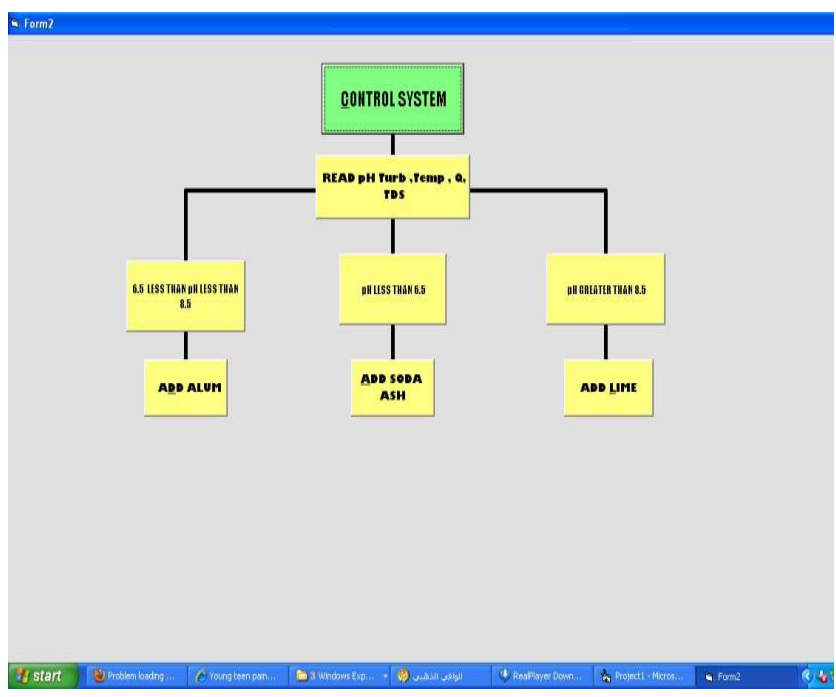

Fig.4: Screen shot of program interface

\section{Tests and Examples}

A sample application of using the software is shown below. Screen shots of Figs.5 and 6 are taken during program implementation and tests.

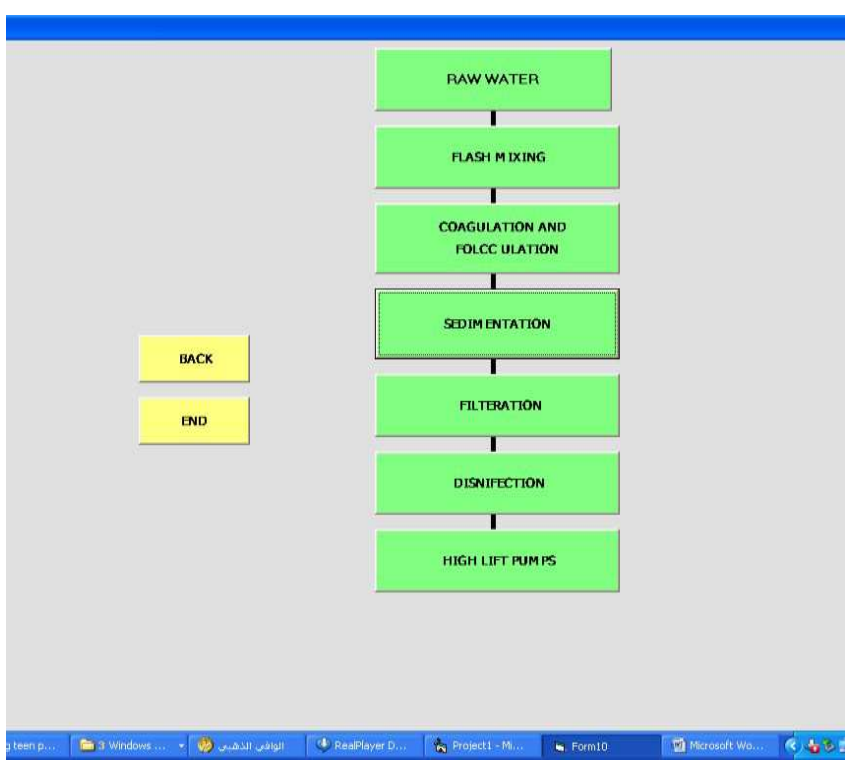

Fig.5: Screen shot of process information page.

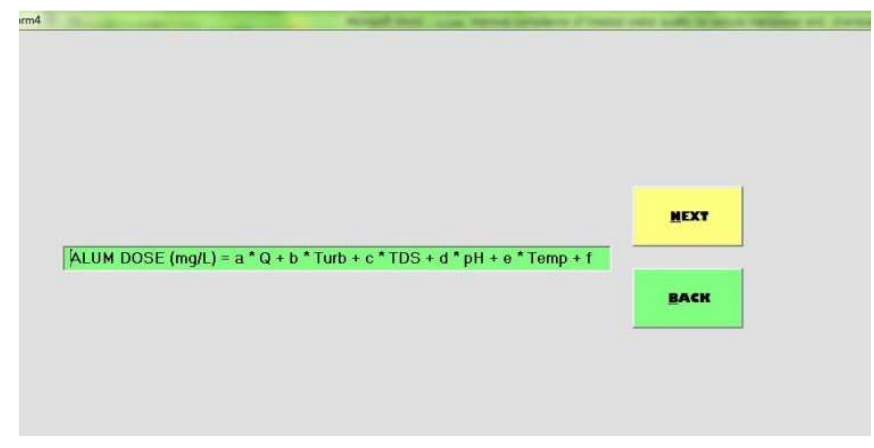

Fig.6: Screen shot of alum dosing information window.

\section{Conclusions}

The aim of this model is to provide water treatment operators with a tool that enables prediction of chemical reagents and treatment conditions for selected removal of turbidity, based on raw water quality data. It was concluded that this system is very powerful tool in improving compliance of treated water quality to reduce manpower and chemicals. The advantages of software design are significant in the water treatment plant operation. The program was designed as an aid, so the user can still customize and optimize the computer suggested design. Users are able to move forward in adjusting or optimizing the design in minutes, which is difficult for manual design. This system was an initial design many new features and functions are to be added to the program to enhance the functions and make it commercially robust.

This is the underlying concept behind the development of this work and to facilitate the organizations and individuals with better understanding on how their actions can have a direct impact on the treatment.

Experimental results using real data have demonstrated the efficiency and soundness of this approach. Significant savings in coagulant usage can be obtained in certain cases. The performance of the system is obviously dependent on the quality and completeness of the data available for training the system. Consequently, continuous updating of training data during operational use is expected to improve the performance of the system. This model, however, is only based on the previous behavior of operators and jartest results. Further work is needed to develop a model taking into account the dynamics of the process, and allowing predicting treated water parameters at the output of the clarification process.

\section{Recommendations}

\section{The following are the recommendations to carry out the computer program:}

- It is important to convince management and technicians with economic data on the benefits of the optimization processes, so that they will implement the recommendations.

- Time and resources have to be allotted for the initial 
and end measurement of the most important indicators in order to be able to compare them.

\section{The following are the recommendations for the program's} user:

- The user should evaluate its own opportunities using the options proposed in this study.

- They should implement, in the following order, the recommendations: 1) reduction techniques at the source, 2) recovery techniques, and 3) treatment technologies.

- Management and employees should think of the chemical reduction processes as continuous, flexible, dynamic, and an integral processes, and not as isolated, rigid, static, and independent activities.

\section{Appendix. Pages 1 and 2 of the Program Software}

\begin{tabular}{|c|c|c|c|c|}
\hline Option Explicit & Option Explicit & MSFlexGrid1.Row $=0$ & MSFlexGrid1.Text = " " & MSFlexGrid1.Col = 3 \\
\hline Private Sub & Private Sub & MSFlexGrid1.Text = " & MSFlexGrid1.Col $=1$ & MSFlexGrid1. Row $=4$ \\
\hline Command1_Click)( & Command1_Click() & Alum, mg/L " & MSFlexGrid1. Row $=7$ & MSFlexGrid1.Text = " " \\
\hline End Sub & Text1.Text $=$ "SODA & MSFlexGrid1.Col $=0$ & MSFlexGrid1.Text = " " & MSFlexGrid1.Col = 3 \\
\hline Private Sub & $\mathrm{ASH}(\mathrm{mg} / \mathrm{L})=\mathrm{m}+\mathrm{n}$ & MSFlexGrid1. Row $=1$ & MSFlexGrid1.Col = 1 & MSFlexGrid1. Row = 5 \\
\hline Command2_Click $)($ & *pH End Sub & MSFlexGrid1.Text = " " & MSFlexGrid1. Row $=8$ & MSFlexGrid1.Text = " " \\
\hline Form 1. Visible $=$ False & Private Sub & MSFlexGrid1.Col $=0$ & MSFlexGrid1.Text = " " & MSFlexGrid1.Col $=3$ \\
\hline Form 2. Visible $=$ True & Command2_Click() & MSFlexGrid1. Row $=2$ & MSFlexGrid1.Col = 1 & MSFlexGrid1. Row = 6 \\
\hline End Sub & Form 4. Visible $=$ False & MSFlexGrid1.Text = "'" & MSFlexGrid1. Row $=9$ & MSFlexGrid1. Text = " " \\
\hline Option Explicit & Form 2. Visible $=$ True & MSFlexGrid1.Col $=0$ & MSFlexGrid1.Text = " " & MSFlexGrid1.Col $=3$ \\
\hline Private Sub & End Sub & MSFlexGrid1 Row $=3$ & MSFlexGrid1.Col $=2$ & MSFlexGrid1. Row $=7$ \\
\hline Command1_Click() & Private Sub & MSFlexGrid1.Text = " " & MSFlexGrid1 Row $=0$ & MSFlexGrid1.Text = " " \\
\hline Form2. Visible $=$ False & Command3_Click() & MSFlexGrid1.Col $=0$ & MSFlexGrid1.Text = "Temp, & MSFlexGrid1 $. \mathrm{Col}=3$ \\
\hline Form9. Visible $=$ True & Form 4. Visible $=$ False & MSFlexGrid1. Row $=4$ & $\mathrm{C}^{\prime \prime}$ & MSFlexGrid1. Row $=8$ \\
\hline End Sub & Form 10. Visible $=$ True & MSFlexGrid1.Text = " " & MSFlexGrid1. Col $=2$ & MSFlexGrid1.Text = " " \\
\hline Private Sub & End Sub & MSFlexGrid1.Col $=0$ & MSFlexGrid1. Row $=1$ & MSFlexGrid1.Col $=3$ \\
\hline Command2_Click(Index & Option Explicit & MSFlexGrid1. Row $=5$ & MSFlexGrid1.Text = " " & MSFlexGrid1. Row $=9$ \\
\hline As Integer) & Private Sub & MSFlexGrid1.Text = " " & MSFlexGrid1.Col = 2 & MSFlexGrid1. Text = " " \\
\hline Form2. Visible $=$ False & Command1_Click() & MSFlexGrid1.Col $=0$ & MSFlexGrid1 Row $=2$ & MSFlexGrid1.Col $=3$ \\
\hline Form 4. Visible $=$ True & Text1.Text = "LIME & MSFlexGrid1 Row $=6$ & MSFlexGrid1.Text = " " & MSFlexGrid1. Row $=3$ \\
\hline End Sub & $\operatorname{DOSE}=\mathrm{j}+\mathrm{k} * \mathrm{pH}$ & MSFlexGrid1.Text = "u" & MSFlexGrid1.Col $=2$ & MSFlexGrid1. Text = " " \\
\hline Private Sub & End Sub & MSFlexGrid1.Col $=0$ & MSFlexGrid1.Row $=3$ & MSFlexGrid1.Col $=4$ \\
\hline Command3_Click() & Private Sub & MSFlexGrid1 Row $=7$ & MSFlexGrid1.Text = " " & MSFlexGrid1. Row $=0$ \\
\hline
\end{tabular}




\begin{tabular}{|c|c|c|c|c|}
\hline Form2. Visible $=$ False & Command10_Click() & MSFlexGrid1.Text = " " & MSFlexGrid1.Col $=2$ & MSFlexGrid1.Text $=$ \\
\hline Form3. Visible $=$ True & Form5 Visible $=$ False & MSFlexGrid1.Col $=0$ & MSFlexGrid1. Row $=4$ & "TDS, mg/L" \\
\hline End Sub & Form 2. Visible $=$ True & MSFlexGrid1. Row $=8$ & MSFlexGrid1.Text = " " & MSFlexGrid1.Col $=5$ \\
\hline Private Sub & End Sub & MSFlexGrid1.Text = " " & MSFlexGrid1.Col $=2$ & MSFlexGrid1.Row $=0$ \\
\hline Command4_Click() & Private Sub & MSFlexGrid1.Col $=0$ & MSFlexGrid1. Row $=5$ & mmS" \\
\hline Form 2 . Visible $=$ False & Command9_Click() & MSFlexGrid1.Row $=9$ & MSFlexGrid1.Text = " " & End Sub \\
\hline Form5. Visible $=$ True & Form5 Visible $=$ False & MSFlexGrid1.Text = " " & MSFlexGrid $1 . \mathrm{Col}=2$ & Option Explicit \\
\hline End Sub & Form 4 .Visible $=$ True & MSFlexGrid1 $. \mathrm{Col}=1$ & MSFlexGrid1.Row $=6$ & Private Sub \\
\hline Option Explicit & End Sub & MSFlexGrid1. Row $=0$ & MSFlexGrid1.Text = " " & Command8_Click() \\
\hline Private Sub & Option Explicit & MSFlexGrid1.Text = & MSFlexGrid1.Col = 2 & End \\
\hline Command1_Click() & Private Sub & "Turb, NTU " & MSFlexGrid1.Row $=7$ & End Sub \\
\hline Text1.Text = "ALUM & Command1_Click() & MSFlexGrid1.Col = 1 & MSFlexGrid1.Text = " " & Private Sub \\
\hline $\begin{array}{l}\text { DOSE }(\mathrm{mg} / \mathrm{L})=\mathrm{a} * \mathrm{Q}+ \\
\mathrm{b}^{*} \mathrm{Turb}+\mathrm{c} \text { TDS } \\
\mathrm{d}^{*} \mathrm{pH}+\mathrm{e}^{*} \mathrm{Temp}+\mathrm{f}\end{array}$ & Form9. Visible $=$ False & MSFlexGrid1.Row $=1$ & MSFlexGrid1.Col $=2$ & Command9_Click() \\
\hline End Sub & Form2. Visible $=$ True & MSFlexGrid1.Text = " " & MSFlexGrid1.Row $=8$ & Form10.Visible $=$ False \\
\hline Private Sub & End Sub & MSFlexGrid1.Col = 1 & MSFlexGrid1.Text = " " & Form 4 .Visible $=$ True \\
\hline Command5_Click() & Private Sub & MSFlexGrid1.Row $=2$ & MSFlexGrid1.Col $=2$ & End Sub \\
\hline Form 3 . Visible $=$ False & Command2_Click() & MSFlexGrid1.Text = " " & MSFlexGrid1.Row $=9$ & \\
\hline Form 4 .Visible $=$ True & Form9. Visible $=$ False & MSFlexGrid1 $. \mathrm{Col}=1$ & MSFlexGrid1.Text = " " & \\
\hline End Sub & Form 4 .Visible $=$ True & MSFlexGrid1.Row $=3$ & MSFlexGrid1.Col $=3$ & \\
\hline \multirow[t]{3}{*}{ Private Sub } & End Sub & MSFlexGrid1.Text = " " & MSFlexGrid1. Row $=0$ & \\
\hline & Private Sub & MSFlexGrid1.Col = 1 & MSFlexGrid1.Text = "pH " & \\
\hline & & MSFlexGrid1.Row $=4$ & & \\
\hline
\end{tabular}

\section{References}

[1] Adgar, A. and Cox, C.S., 1997,"Process fault detection and reconstruction using statistical and advanced techniques: Some preliminary findings", Proc. Int. Conf. System Engineering, Coventry, UK, September.

[2] Babylon Water Directorate, 2012.

[3] Bernazeau, F., Pierrone, P., and. Duguet, J.P, 1992,"Interest in using a streamline current detector for automatic coagulant dose control, Journal of Water Supply.

[4] Chang, M., 2002,"Forest Hydrology: An Introduction to Water and Forests", CRC Press, Boca Raton, Florida.
[5] Cox, C.S. and Graham, J., 1994,"Steps towards automatic clarification control", IEE Colloq. Advances in control in the process industries: Computing and control division, London, UK, March.

[6] Dentel, K.S, 1995,"Use of streaming current detector in coagulation monitoring and control", Journal of Water Sciences Research and Technologies - Aqua. 44 .

[7] Evans, J., Enoch, C., Johnson M., and Williams, P., 1998,"Intelligent based autocoagulation control applied to a water treatment works, in: Proceedings of International Conference on Control.

[8] Fu-Yi Cui, Yuan-zhen, 1999, "Water Supply and Sewerage Project Instrumentation and Control. Beijing: China Building Industry Press. 
[9] Grimm, J. W., and Lynch, J. A., 2004,"Enhanced wet deposition estimates using modeled precipitation inputs", Environmental Monitoring and Assessment, vol. 90, No. 1-3.

[10] Kenichi Kurotfanim, et al., 1995, "Advanced control of coagulation process applying floc sensor", In: IWSA. Specialized Conference on Advanced Treatment and Intergrated Water System Management into the 21st Century.

[11] Lind, C., 1994,"Coagulation Control and Optimization", Part One and Part Two, Public Works (October).

[12] Mirsepassi, A., Cathers B., and Dharmappa, H.B., 1995,"Application of Artificial Neural Networks to the Real Time Operation of Water Treatment Plants, in: Proceedings of the International Conference on Neural Networks, (Vol. 1), Perth, Australia.

[13] Prakash, A., 2004,"Water Resources Engineering", ASCE Press, New York.
[14] Song Qi-min, Lumin Gang, Yi Yong, et al, 1999, "Automatic control of the amount of coagulant filling new method. China Water \&amp; Wastewater.

[15] Yang people, 2000, "Display type flocculation control system (FCD) in the water treatment plant applications", China Water \&amp; Wastewater.

[16] Zhang Weiguo, 2000, "Introduction of advanced control theory and methods", Xi'an: Northwest Industry University Press,. Reposted elsewhere in the Research PapersDownloadhttp://www.hi138.com

[17] Zhang, W. L., Wu, S. X. and Ji, H. J., 2007,"Estimation of agricultural non-point source pollution in China and the alleviating strategies", Scientia Agricultura Sinica, vol. 37, no 7.

[18] Zhong Chun-chang, et al., 1989, "Mathematical model plus alum automation technologies. China Water Supply and Drainage. 